\title{
DETERMINACIÓN DE VITAMINA C, COMPUESTOS FENÓLICOS TOTALES Y ACTIVIDAD ANTIOXIDANTE DE FRUTAS DE GUAYABA (Psidium guajava L.) CULTIVADAS EN COLOMBIA
}

\author{
Dayana Rojas-Barquera \\ Facultad de Ciencias y Tecnología, Universidad Mayor de San Simón, Cochabamba, Bolivia \\ Carlos-Eduardo Narváez-Cuenca* \\ Cra $30 N^{\circ} 45-03$ \\ Recebido em 19/11/08; aceito em 15/5/09; publicado na web em 13/10/09
}

Departamento de Química, Facultad de Ciencias, Universidad Nacional de Colombia, Ciudad Universitaria, Bogotá - Colombia

\begin{abstract}
VITAMIN C, TOTAL PHENOLIC COMPOUNDS AND ANTIOXIDANT ACTIVITY IN GUAVA (Psidium guajava L.) FRUITS FROM COLOMBIA. Some physicochemical parameters, vitamin C, total phenolic compounds and antioxidant activity (AA) measured by ABTS, FRAP and DPPH methods were determinated in four different varieties of ripe guava produced in Colombia. Samples were statistically similar in their titratable acidity. Soluble solids were statistically similar and higher in "Pear", "Pink Regional", and "White Regional", but lower in "Apple" guavas. Vitamin C was statistically lower in "Pear" guava. Phenolics, ABTS-, FRAP-, and DPPH-AA were statistically lower in "Apple" guava if compared in wet basis. "Pink Regional" and "White Regional" contained the highest levels in vitamin $\mathrm{C}$, phenolics and antioxidant activity.
\end{abstract}

Keywords: DPPH; ascorbic acid; Psidium guajava.

\section{INTRODUCCIÓN}

En Colombia se cultivan diferentes variedades de guayaba dentro de las que se incluyen la "Regional Roja", "Regional Blanca", "Pera" y "Manzana". En su estado maduro estas frutas tienen corteza amarilla y pulpa rosada, corteza amarilla y pulpa amarilla, corteza amarilla y pulpa roja y corteza verde y pulpa blanca, respectivamente. Este tipo de fruto se consume en fresco y en preparaciones como néctar, jalea, bocadillo, entre otros. La producción de guayaba se concentra en los departamentos de Santander y Boyacá (60\% del área del país), Tolima (10\%), Cundinamarca (9\%), Huila, Antioquia, Cauca, Nariño y Atlántico, principalmente.

El estudio de antioxidantes naturales ha cobrado un papel importante como resultado de su relación directa con la disminución del riesgo a sufrir enfermedades coronarias y cáncer, entre otras. ${ }^{1}$ Compuestos presentes en frutas y hortalizas como tocoferoles, ácidos fenólicos, flavonoides, vitamina $\mathrm{C}$ y carotenoides han sido vinculados a efectos positivos en la salud por su acción antioxidante. ${ }^{1}$ Diversas investigaciones muestran que los frutos de guayaba tienen aportes importantes en vitamina $\mathrm{C}$, en compuestos fenólicos y que su ingesta puede contribuir a una adecuada actividad antioxidante..$^{2-7}$ De otro lado, es claro que tanto el contenido de este tipo de sustancias, como la actividad antioxidante pueden ser diferentes dependiendo de la variedad de guayaba en estudio. ${ }^{4-7}$

Si se tiene en cuenta que tanto el ácido L-ascórbico como el ácido L-dehidroascórbico tienen actividad biológica como vitamina C la técnica HPLC es adecuada para cuantificar ambas formas vitamínicas. ${ }^{8,9}$ En la técnica el ácido dehidroascórbico es convertido en ácido ascórbico mediante una reacción redox con ditiotreitol. De otro lado, la técnica de medida de compuestos fenólicos totales es relativamente fácil y requiere equipos poco sofisticados; aunque es ampliamente usada, tiene como limitante que otros compuestos pueden dar reacción positiva, por lo que se considera que su valor tiende a ser sobreestimado. ${ }^{10}$

Además de la cuantificación por separado de compuestos que pueden contribuir a la actividad antioxidante, se recurre también a la medida

*e-mail: cenarvaezc@unal.edu.co de la actividad antioxidante del conjunto de compuestos antioxidantes, mediante la extracción en diferentes tipos de solvente y medida de su actividad por ciertas técnicas. Los antioxidantes pueden actuar de manera diferente dependiendo no sólo de su composición química sino también de la fuente de radicales libres o de agentes oxidantes, por lo que se van a obtener diferentes respuestas dependiendo de la técnica empleada. ${ }^{10}$ Los autores de la referencia dan una clara explicación sobre las ventajas y desventajas de cada técnica. Así por ejemplo, los métodos FRAP y ABTS se basan en una reacción redox con potenciales menores a 0,7 $\mathrm{V}$, por lo que representa la situación celular; son rápidos, luego de 4 y 6 min respectivamente se obtienen medidas estables para patrones pero, para muestras reales se debe monitorear la reacción durante un mayor tiempo puesto que algunos polifenoles reaccionan lentamente. La técnica DPPH es rápida y sencilla, por lo que es también ampliamente usada; sin embargo otras sustancias que absorban a $515 \mathrm{~nm}$ pueden interferir.

Es importante conocer el aporte de vitamina $\mathrm{C}$ y la actividad antioxidante de alimentos que hacen parte de la dieta de un grupo poblacional para garantizar a través de la ingesta un consumo lo más adecuado posible de sustancias con interés nutricional. El objetivo de este trabajo fue evaluar el contenido de vitamina C, ácido ascórbico, ácido dehidroascórbico, fenoles totales y actividad antioxidante, medida por ABTS, FRAP y DPPH, en cuatro variedades diferentes de guayaba (Psidium guajava L.) cultivadas en Colombia.

\section{PARTE EXPERIMENTAL}

\section{Reactivos}

Los reactivos TPTZ [2, 4, 6-(tri-(2-piridil-s-triazina))], ABTS (2,2'-azinobis (3-etilbenzotiazolina-6-ácido sulfónico)), DPPH (radical 1,1-difenil-2-picrilhidrazilo) y DL-Ditiotreitol (DTT) fueron adquiridos de SIGMA. El reactivo Trolox (ácido-6-hidroxi-2, 5, 7, 8 tetrametilcromano-2-carboxílico) fue adquirido de Aldrich. El reactivo de Folin Ciocalteu fue adquirido de Panreac Química S. A. El ácido ascórbico $\left(\mathrm{C}_{6} \mathrm{H}_{8} \mathrm{O}_{6}\right)$ y el fosfato monobásico de potasio $\left(\mathrm{KH}_{2} \mathrm{PO}_{4}\right)$ de Merck, el ácido gálico monohidratado $\left(\mathrm{C}_{6} \mathrm{H}_{2}(\mathrm{OH})_{3} \mathrm{COOH} . \mathrm{H}_{2} \mathrm{O}\right)$ de J.T. Baker. Los otros reactivos que se emplearon fueron de Merck. 


\section{Muestras de guayaba}

Las muestras de guayaba "Regional Roja" y "Regional Blanca" provinieron del municipio de Vélez (Santander). Las frutas fueron cosechadas, enviadas inmediatamente a Bogotá, a la Universidad Nacional de Colombia y analizadas. El tiempo transcurrido entre la recolección y el análisis fue de 2 días a $18{ }^{\circ} \mathrm{C}$. Las guayabas "Pera" y "Manzana" se obtuvieron de supermercados ubicados en la ciudad de Bogotá, con almacenamiento previo de entre 0 a 3 días a $18{ }^{\circ} \mathrm{C}$. Las muestras fueron obtenidas entre los meses de julio a octubre de 2008. Se seleccionaron muestras en estado maduro. Se obtuvieron cuatro lotes diferentes de cada variedad, cada uno adquirido en tiempo diferente o de lugares diferentes. Cada lote estaba constituido por 20 frutos.

\section{Análisis fisicoquímicos}

Se tomaron al azar mínimo 3 frutos de cada lote y se midió en cada fruto el diámetro longitudinal y transversal con un calibrador, el peso fresco en balanza semianalítica y la dureza $\left(\mathrm{Kgf} / \mathrm{cm}^{2}\right)$ mediante un penetrómetro. Posteriormente, las 20 frutas de cada lote fueron troceadas y los trozos resultantes (aproximadamente $3 \times 3 \mathrm{~cm}$ ) fueron mezclados. Se efectuó un cuarteo, la mitad de la muestra se desechó y la otra mitad se despulpó de manera manual. Las semillas fueron retiradas y el conjunto corteza más pulpa fue homogenizado para obtener un puré. Sobre la fracción comestible se midió la acidez titulable, el contenido de sólidos solubles, el pH y la humedad. Se calculó además, el índice de madurez ( ${ }^{\circ}$ Brix/acidez). La acidez titulable se evaluó mediante titulación con $\mathrm{NaOH} 0,1 \mathrm{M}$ y se expresó como \% ácido ascórbico. Los sólidos solubles se midieron por refractometría y se espresaron como ${ }^{\circ}$ Brix. El pH se midió con un pH-metro. El contenido de humedad se midió mediante gravimetría por secado a $70{ }^{\circ} \mathrm{C}$ durante $24 \mathrm{~h}$.

\section{Ácido ascórbico, dehidroascórbico y vitamina C}

Los contenidos de ácido ascórbico, dehidroascórbico y vitamina C fueron medidos el mismo día en que se efectuó el despulpado. Se empleó la técnica HPLC. ${ }^{8}$ Un volumen de $20 \mu \mathrm{L}$ de muestra o de patrón fue inyectado a una columna en acero inoxidable, RP-18, Licrhosorb, $5 \mu \mathrm{m}$, operada a temperatura ambiente. La fase móvil fue $\mathrm{KH}_{2} \mathrm{PO}_{4} 0,2 \mathrm{M}$ ajustado a $\mathrm{pH} 2,4$ con $\mathrm{H}_{3} \mathrm{PO}_{4}$ a un flujo de 0,5 $\mathrm{mL} / \mathrm{min}$. Se utilizó un detector UV-vis a $254 \mathrm{~nm}$ a $30^{\circ} \mathrm{C}$. La curva de calibración se construyó con ácido ascórbico a concentraciones entre 10 a $50 \mathrm{mg} / \mathrm{L}$, con $1 \mathrm{mg} / \mathrm{mL}$ de DTT. Para la extracción de la vitamina $\mathrm{C}$ de las frutas de guayaba se pesaron $2,0 \mathrm{~g}$ del puré y se extrajeron con $8,0 \mathrm{~mL}$ de agua grado HPLC mediante agitación magnética durante $5 \mathrm{~min}$. Los extractos fueron centrifugados a 1.493 $\mathrm{X}$ g durante $15 \mathrm{~min}$ a $4{ }^{\circ} \mathrm{C}$. El sobrenadante fue filtrado a través de un filtro Millipore de 0,45 $\mu \mathrm{m}$. Para cuantificar el ácido ascórbico se inyectó el extracto previamente diluido con agua tipo HPLC para que la lectura cayera dentro de la curva de calibración. Para cuantificar la vitamina C (ácido ascórbico + ácido dehidroascórbico) se tomó 1 $\mathrm{mL}$ del extracto previamente diluido y se le incorporó $1 \mathrm{mg}$ de DTT. La mezcla de dejó reaccionar durante 2 h en la oscuridad y se inyectó al HPLC. El ácido dehidroascórbico se calculó por diferencia entre el contenido total de vitamina $\mathrm{C}$ y el ácido ascórbico.

\section{Compuestos fenólicos totales y actividad antioxidante}

La obtención de los extractos para la medición de fenoles totales y actividad antioxidante fue realizada luego de un día de almacenamiento del puré a $-20^{\circ} \mathrm{C}$. Se pesaron $0,5 \mathrm{~g}$ de fracción comestible ho- mogenizada y se le agregaron $10,0 \mathrm{~mL}$ de una solución metanol:agua (50:50). La mezcla fue agitada mediante agitador magnético a $50{ }^{\circ} \mathrm{C}$ durante $30 \mathrm{~min}$. Posteriormente se centrifugó a $1.493 \mathrm{X}$ g durante 15 min a $4{ }^{\circ} \mathrm{C}$. El sobrenadante se almacenó en la oscuridad a $-20{ }^{\circ} \mathrm{C}$. El residuo fue sometido a una segunda extracción con $10,0 \mathrm{~mL}$ de acetona:agua (70:30) a $50{ }^{\circ} \mathrm{C}$ por 30 min y se centrifugó a $1.493 \mathrm{X}$ g por 15 min a $4^{\circ} \mathrm{C}$. En total se efectuaron cuatro extracciones con acetona:agua (70:30). Los cinco sobrenadantes fueron reunidos y se llevó a $50 \mathrm{~mL}$ con agua destilada. Finalmente, se efectuó la cuantificación de fenoles totales y de actividad antioxidante.

\section{Compuestos fenólicos totales}

La concentración de fenoles totales se midió con el método de Folin-Ciocalteu. ${ }^{11} \mathrm{Un}$ volumen de $20 \mu \mathrm{L}$ de los extractos se mezcló con $1580 \mu \mathrm{L}$ de agua y $100 \mu \mathrm{L}$ de una solución de Folin-Ciocalteu sin dilución previa. La mezcla se dejó en reposo por $5 \mathrm{~min}$. Posteriormente, se adicionaron $300 \mu \mathrm{L}$ de una solución de carbonato de sodio al 20\%. Después de $2 \mathrm{~h}$ de reacción se midió la absorbancia a $765 \mathrm{~nm}$. Se efectuaron curvas de calibración con ácido gálico a concentraciones entre 50 a $500 \mathrm{mg} / \mathrm{L}$. Los resultados se expresaron como equivalentes de ácido gálico (mg ácido gálico/100 g fracción comestible).

\section{Capacidad antioxidante}

Se emplearon los métodos de ABTS, ${ }^{12} \mathrm{FRAP}^{13}$ y DPPH. ${ }^{14}$

Para el método de ABTS se preparó una solución compuesta por ABTS $7 \mathrm{mM}$ y persulfato de potasio $0,45 \mathrm{mM}$. La mezcla se dejó reposar en la oscuridad durante $16 \mathrm{~h}$ a temperatura ambiente. La solución stock se almacenó a $-20{ }^{\circ} \mathrm{C}$ en la oscuridad. Cada vez que era necesario se diluía la solución stock con etanol hasta alcanzar una absorbancia de 0,7 $\pm 0,02$ a $734 \mathrm{~nm}$. Se prepararon curvas de calibración con Trolox a concentraciones entre 250 a $1500 \mu \mathrm{M}$. Se tomaron $1000 \mu \mathrm{L}$ del reactivo catión radical $\mathrm{ABTS}^{+}$y se incubó a 30 ${ }^{\circ} \mathrm{C}$. Una vez alcanzada la temperatura de incubación se adicionaron 10 $\mu \mathrm{l}$ de soluciones de Trolox. La absorbancia de la mezcla de reacción fue monitoreada a $734 \mathrm{~nm}$ cada $5 \mathrm{~s}$ durante $6 \min$ a $30{ }^{\circ} \mathrm{C}$. Para los extractos de guayaba se realizaron diluciones y en lugar de los 10 $\mu \mathrm{L}$ de Trolox se adicionaron $10 \mu \mathrm{L}$ de extracto previamente diluido. Para los extractos fue necesario un tiempo de 45 min para obtener una medida estable en la absorbancia. Los resultados fueron expresados en $\mu \mathrm{mol}$ Trolox/g fracción comestible.

Para el método FRAP se preparó siempre el reactivo fresco constituido por $25 \mathrm{~mL}$ de buffer acetato de sodio $300 \mathrm{mM}$ a pH 3,6, 2,5 mL de TPTZ $10 \mathrm{mM}$ disuelto en $\mathrm{HCl} 40 \mathrm{mM}$ y $2,5 \mathrm{~mL}$ de $\mathrm{FeCl}_{3} \cdot 6 \mathrm{H}_{2} \mathrm{O}$ $20 \mathrm{mM}$. Se prepararon curvas de calibración con soluciones de Trolox con concentraciones entre 100 a $1500 \mu \mathrm{M}$. Se tomaron $900 \mu \mathrm{L}$ del reactivo de FRAP, se adicionaron $90 \mu \mathrm{L}$ de agua y se incubó a $37{ }^{\circ} \mathrm{C}$. Una vez alcanzada la temperatura de incubación se adicionaron $30 \mu \mathrm{L}$ de solución de Trolox. Se monitoreó la absorbancia a $593 \mathrm{~nm}$ cada 5 seg durante 4 min. Para las muestras se realizaron diluciones adecuadas y su actividad se midió de igual forma que en los patrones, excepto que el tiempo de incubación necesario para llegar a la máxima respuesta fue de $2 \mathrm{~h}$. Los resultados fueron expresados en $\mu \mathrm{mol}$ Trolox/g fracción comestible.

Para el método DPPH se preparó una solución stock de DPPH 0,6 mM en metanol y se almacenó a $-20^{\circ} \mathrm{C}$ en la oscuridad. Cada vez que era necesario se preparaba una solución de trabajo por dilución de la solución stock con metanol hasta obtener una absorbancia de 1,1 \pm 0,02 a $515 \mathrm{~nm}$. Se prepararon curvas de calibración con soluciones de Trolox con concentraciones entre 100 a $1500 \mu \mathrm{M}$. Se tomaron 975 $\mu \mathrm{L}$ del reactivo de DPPH a temperatura ambiente. Se adicionaron 
$25 \mu \mathrm{L}$ de solución de Trolox. Se monitoreó la absorbancia a $515 \mathrm{~nm}$ cada 5 seg durante $7 \mathrm{~min}$. La actividad antioxidante de las muestras se midió de igual forma que en los patrones, excepto que el tiempo total de medida fue de $20 \mathrm{~min}$. Los resultados fueron expresados en $\mu \mathrm{mol}$ Trolox/g fracción comestible.

\section{Análisis estadístico}

Las medidas de diámetro longitudinal, transversal, peso fresco y dureza se efectuaron sobre un total de mínimo 13 frutos diferentes. Las medidas de sólidos solubles, $\mathrm{pH}$, acidez, humedad, vitamina $\mathrm{C}$ y actividad antioxidante se efectuaron sobre 4 lotes diferentes de la fracción comestible de guayaba, con dos replicaciones. Sobre las variables de respuesta se determinaron los promedios y las desviaciones estándar. Se realizaron ANOVAS y se aplicó la prueba Tukey para determinar las diferencias entre variedades de guayaba $(\mathrm{p}<0,05)$. Se calcularon los coeficientes de correlación entre las variables de respuesta de interés por la prueba de Pearson. Los análisis fueron efectuados a través del programa Statgraphics 5,1 plus ${ }^{\circledR}$.

\section{RESULTADOS Y DISCUSIÓN}

En la Tabla 1 se consigna información que describe algunas características generales de las frutas en estudio. La guayaba variedad "Manzana" fue la de mayor tamaño (diámetro transversal y longitudinal) y mayor peso por unidad que las otras guayabas en estudio, si bien en su diámetro longitudinal no hubo diferencias significativas entre las guayabas "Manzana" y "Pera". Les siguieron en orden descendente la variedad "Regional Roja" y "Regional Blanca". La dureza fue estadísticamente mayor en la variedad "Manzana". Los mayores contenidos de humedad se encontraron para las variedades "Manzana" y "Pera". De las frutas en estudio, la variedad "Regional Blanca" tuvo los máximos valores de sólidos solubles, si bien los valores fueron estadísticamente iguales a los encontrados en las variedades "Regional Blanca" y "Pera"; los menores valores de sólidos solubles se presentaron en la guayaba "Manzana". No hubo diferencias estadísticas ni en la acidez ni en el índice de madurez ( ${ }^{\circ}$ Brix/acidez). Sin embargo, la variedad "Regional Blanca" fue la más ácida del conjunto y la variedad "Manzana" la menos ácida. Aunque el índice de madurez fue mayor en la variedad "Manzana" es claro que ésta fue menos dulce y menos ácida que las otras.

\section{Contenido de vitamina $\mathrm{C}$, fenoles totales y actividad antioxidante}

Los resultados obtenidos para vitamina $\mathrm{C}$ en las cuatro variedades de guayaba se muestran en la Tabla 2. También se calcularon los valores en base seca para facilitar la comparación con otros reportes. En todos los casos, la contribución del ácido ascórbico al contenido de vitamina $\mathrm{C}$ fue mayor que el aporte del ácido dehidroascórbico. El mayor contenido de vitamina $\mathrm{C}$, tanto en base húmeda como en base seca, se obtuvo para la guayaba "Regional Roja", si bien los niveles en la "Regional Blanca" y "Manzana" fueron estadísticamente iguales al de la "Regional Roja". El contenido de vitamina C en la variedad "Pera" fue estadísticamente inferior al de las otras variedades. La contribución del ácido dehidroascórbico en los niveles de vitamina $\mathrm{C}$ fue mayor en la guayaba "Pera" $(33,2 \%)$ y menor en la "Regional Blanca" y "Manzana" (17,1 y 16,1\%, respectivamente). Existen reportes en los que se indica que la contribución del ácido dehidroascórbico a la vitamina $C$ varía entre 1,6 hasta $40,6 \%$ para frutas y entre 71,9 hasta $100 \%$ para hortalizas. ${ }^{8}$ De otro lado, para la fracción comestible, constituida por pulpa más corteza, se reportan valores de ácido ascórbico que varían ampliamente, entre 74 a 84 para guayaba de pulpa rosada cultivada en Ecuador hasta $397 \pm 25$ mg/100 g B.H. para guayaba de pulpa rosada, genotipo Fan Retief, cultivada en Estados Unidos. ${ }^{3,4}$

El contenido de fenoles totales, en base húmeda, fue mayor en las variedades "Regional Roja" y "Regional Blanca" e inferior en las variedades "Pera" y "Manzana". Sin embargo, dada la alta dispersión entre muestras obtenida para la guayaba "Pera", sus niveles fueron estadísticamente iguales a los de las variedades "Regional Roja" y "Regional Blanca". Al efectuar la comparación del contenido de fenoles en base seca no se encontraron diferencias estadísticas entre las cuatro variedades. Se encontraron las mayores actividades antioxidantes, medidas por los métodos ABTS, FRAP y DPPH, para las variedades "Pera", "Regional Roja" y "Regional Blanca", en tanto las respuestas para la variedad "Manzana" fueron las menores del conjunto. El análisis estadístico indicó que la actividad antioxidante medida por los métodos ABTS y FRAP fue significativamente inferior en la guayaba "Manzana" en base húmeda; lo anterior también se obtuvo para los resultados expresados en base seca en el método FRAP. De otro lado, en el método DPPH se encontraron dos agrupaciones: en base húmeda las guayabas "Pera", "Regional Roja" y "Regional Blanca" tuvieron una respuesta estadísticamente igual; en tanto que

Tabla 1. Propiedades fisicoquímicas de guayabas (Psidium guajava L.) de las variedades "Pera", "Regional Roja", "Regional Blanca” y "Manzana"

\begin{tabular}{|c|c|c|c|c|}
\hline & \multicolumn{4}{|c|}{ Variedad } \\
\hline & Pera & $\begin{array}{c}\text { Regional } \\
\text { Roja }\end{array}$ & Regional Blanca & Manzana \\
\hline Diámetro longitudinal (cm) & $6,6 \pm 2,2 \mathrm{ab}(17)$ & $5,5 \pm 0,9 \mathrm{~b}(13)$ & $3,8 \pm 0,3$ c (13) & $7,0 \pm 0,8$ a $(15)$ \\
\hline Diámetro transversal $(\mathrm{cm})$ & $5,1 \pm 1,6 \mathrm{~b}(17)$ & $4,5 \pm 0,9$ bc $(13)$ & $3,4 \pm 0,3$ c (13) & $8,1 \pm 0,7$ a $(15)$ \\
\hline Peso (g) & $136,7 \pm 52,6 \mathrm{~b}(17)$ & $70,7 \pm 19,7$ c (13) & $60,8 \pm 15,0$ c (13) & $314,8 \pm 76,7$ a (15) \\
\hline Dureza $\left(\mathrm{kgf} / \mathrm{cm}^{2}\right)$ & $6,4 \pm 2,3 \mathrm{~b}(17)$ & $6,2 \pm 1,9 \mathrm{~b}(13)$ & $4,9 \pm 1,7 \mathrm{~b}(13)$ & $8,8 \pm 1,4$ a $(15)$ \\
\hline Humedad (\%) & $87,3 \pm 4,3 \mathrm{ab}(4)$ & $85,8 \pm 0,4 \mathrm{~b}(4)$ & $85,3 \pm 0,5 \mathrm{~b}(4)$ & $91,8 \pm 0,4$ a (4) \\
\hline${ }^{\circ}$ Brix & $7,5 \pm 2,2 \mathrm{ab}(4)$ & $8,6 \pm 0,6$ a (4) & $9,5 \pm 0,4$ a (4) & $5,9 \pm 0,2 \mathrm{~b}(4)$ \\
\hline $\mathrm{pH}$ & $4,0 \pm 0,2$ a (4) & $3,6 \pm 0,0 \mathrm{~b}(4)$ & $3,7 \pm 0,1 \mathrm{~b}(4)$ & $4,0 \pm 0,0$ a (4) \\
\hline Acidez (\% ácido ascórbico) & $0,72 \pm 0,44$ a $(4)$ & $0,79 \pm 0,03$ a (4) & $0,94 \pm 0,23$ a (4) & $0,38 \pm 0,05$ a $(4)$ \\
\hline${ }^{\circ}$ Brix/acidez & $12 \pm 4 \mathrm{a}(4)$ & $11 \pm 1$ a (4) & $10 \pm 2$ a (4) & $16 \pm 2 \mathrm{a}(4)$ \\
\hline
\end{tabular}

Los valores están expresados en base húmeda y se muestran como el promedio $\pm 1 \mathrm{SD}$. Entre paréntesis se indica el número de muestras analizadas. Letras iguales dentro de una fila indican que no hay diferencias significativas de acuerdo a la prueba de Tukey $(\mathrm{P} \leq 0,05)$. 
Tabla 2. Contenido de Vitamina C, fenoles totales y actividad antioxidante en la fracción comestible de guayaba (Psidium guajava L.) en las variedades "Pera", "Regional Roja", "Regional Blanca" y "Manzana"

\begin{tabular}{|c|c|c|c|c|}
\hline & \multicolumn{4}{|c|}{ Variedad } \\
\hline & Pera & Regional Roja & Regional Blanca & Manzana \\
\hline $\mathrm{AA}(\mathrm{mg} / 100 \mathrm{~g})$ & $\begin{array}{c}52,2 \pm 7,6 b \\
(437 \pm 116 b)\end{array}$ & $\begin{array}{l}206,6 \pm 64,6 a \\
(1444 \pm 414 a)\end{array}$ & $\begin{array}{l}179,6 \pm 31,8 a \\
(1222 \pm 210 a)\end{array}$ & $\begin{array}{l}147,2 \pm 13,1 a \\
(1797 \pm 179 a)\end{array}$ \\
\hline $\mathrm{ADHA}(\mathrm{mg} / 100 \mathrm{~g})$ & $\begin{array}{l}26,0 \pm 18,0 b \\
(204 \pm 141 b)\end{array}$ & $\begin{array}{c}62,1 \pm 11,7 a \\
(436 \pm 79 a)\end{array}$ & $\begin{array}{l}37,1 \pm 5,0 a b \\
(253 \pm 41 a b)\end{array}$ & $\begin{array}{c}28,2 \pm 7,2 b \\
(346 \pm 100 a b)\end{array}$ \\
\hline Vitamina C (mg/100g) & $\begin{array}{l}78,2 \pm 15,6 b \\
(641 \pm 121 b)\end{array}$ & $\begin{array}{l}268,7 \pm 73,4 a \\
(1880 \pm 469 a)\end{array}$ & $\begin{array}{l}216,6 \pm 34,1 a \\
(1107 \pm 762 a)\end{array}$ & $\begin{array}{l}175,5 \pm 10,5 a \\
(2143 \pm 193 a)\end{array}$ \\
\hline Fenoles Totales (mg AG/100 g) & $\begin{array}{c}298 \pm 190 a b \\
(2329 \pm 1309 a)\end{array}$ & $\begin{array}{c}508 \pm 47 a \\
(3577 \pm 354 a)\end{array}$ & $\begin{array}{c}496 \pm 99 a \\
(2494 \pm 1341 a)\end{array}$ & $\begin{array}{c}258 \pm 55 b \\
(3167 \pm 804 a)\end{array}$ \\
\hline ABTS ( $\mu$ molTrolox/g) & $\begin{array}{l}95,5 \pm 39,6 a \\
(846 \pm 509 a)\end{array}$ & $\begin{array}{l}107,3 \pm 24,0 a \\
(755 \pm 171 a b)\end{array}$ & $\begin{array}{l}100,1 \pm 10,4 a \\
(682 \pm 69 a b)\end{array}$ & $\begin{array}{l}13,6 \pm 0,7 b \\
(166 \pm 10 b)\end{array}$ \\
\hline FRAP ( $\mu$ molTrolox/g) & $\begin{array}{l}63,1 \pm 28,7 a \\
(492 \pm 107 a)\end{array}$ & $\begin{array}{c}77,0 \pm 11,0 a \\
(541 \pm 73 a)\end{array}$ & $\begin{array}{c}66,2 \pm 10,7 a \\
(450 \pm 57 a)\end{array}$ & $\begin{array}{l}20,8 \pm 4,1 b \\
(252 \pm 42 b)\end{array}$ \\
\hline DPPH ( $\mu$ molTrolox/g) & $\begin{array}{c}25,7 \pm 14,5 a b \\
(195 \pm 50 a)\end{array}$ & $\begin{array}{c}26,1 \pm 6,1 \mathrm{ab} \\
(183 \pm 41 \mathrm{a})\end{array}$ & $\begin{array}{l}32,5 \pm 6,2 \mathrm{a} \\
(221 \pm 40 \mathrm{a})\end{array}$ & $\begin{array}{l}10,8 \pm 0,9 b \\
(131 \pm 15 a)\end{array}$ \\
\hline
\end{tabular}

Los valores están expresados en base húmeda y se muestran como el promedio $\pm 1 \mathrm{SD}(\mathrm{n}=4)$. Letras iguales dentro de una fila indican que no hay diferencias significativas de acuerdo a la prueba de Tukey $(\mathrm{P} \leq 0,05)$. Entre paréntesis aparecen los resultados en base seca. AA: Ácido ascórbico. ADHA: Ácido dehidroascórbico. AG: Ácido gálico.

en base seca no se encontraron diferencias en la actividad medida por DPPH para las cuatro variedades. Los contenidos de fenoles totales obtenidos en este trabajo están en un intervalo entre igual a superior respecto de los reportados por otros autores, en el que hay gran variación: desde 20 hasta $462 \pm 128 \mathrm{mg}$ ácido gálico/100 g. ${ }^{3,5}$ $\mathrm{Al}$ comparar en base seca hay una excepción respecto al reportado para corteza de guayaba cultivada en Venezuela $(5870 \pm 400 \mathrm{mg}$ ácido gálico/100 g) que es mayor al intervalo encontrado en este trabajo para el conjunto corteza más pulpa $(2329 \pm 1309$ a $3577 \pm 354 \mathrm{mg}$ ácido gálico/100 g, para guayaba "Pera" y "Regional Roja", respectivamente). ${ }^{7}$ Sin embargo, si se observa en detalle la referencia del trabajo de Venezuela, aunque la actividad antioxidante es mayor en la corteza ( $5870 \pm 400 \mathrm{mg}$ ácido gálico/100 g), es menor en la pulpa (2630 $\pm 80 \mathrm{mg}$ ácido gálico/100 g). ${ }^{7}$ Las actividades antioxidantes medidas por ABTS y FRAP en las variedades "Pera", "Regional Roja" y "Regional Blanca" fueron superiores a las reportadas en otros estudios. Así, por el método ABTS, se reporta para guayaba de pulpa rosada, Ruby Supreme, cultivada en Estados Unidos, un valor inferior de 22,3 $\pm 0,9 \mu \mathrm{mol}$ Trolox/g parte comestible y para guayaba de pulpa rosada cultivada en Ecuador un valor superior de $46 \pm 11$ $\mu \mathrm{mol}$ Trolox/g parte comestible. ${ }^{3,4}$ Por el método FRAP se reporta para guayaba cultivada en Tailanda, un valor inferior de 15,0 $\pm 0,8 \mu \mathrm{mol}$ Trolox/g parte comestible y un valor superior para guayaba de pulpa blanca, genotipo Allahabad Safeda, cultivada en Estados Unidos, de $33,3 \pm 1,4 \mu \mathrm{mol}$ Trolox/g parte comestible. ${ }^{2,4}$ Para las tres variedades antes mencionadas la actividad por el método DPPH está dentro del intervalo de otras investigaciones; intervalo comprendido entre $16,2 \pm 1,0$ y $32,0 \pm 5,1 \mu \mathrm{mol}$ Trolox/g parte comestible, para guayaba de pulpa rosada, Ruby Supreme y para guayaba de pulpa blanca, genotipo Allahabad Safeda, ambas cultivadas en Estados Unidos, respectivamente. Para guayaba "Manzana" la actividad ABTS y DPPH está por debajo del reportado por otros autores, en tanto que la actividad medida por FRAP está dentro del intervalo. Era de esperarse obtener valores altos para los resultados de fenoles totales y actividad antioxidante como resultado de la aplicación de una técnica de extracción en la que se extraen compuestos de diversa polaridad, al recurrir a la extracción no sólo con metanol sino también con acetona.
Los ensayos de correlaciones entre variables (Tabla 3) dejan ver que el contenido de ácido ascórbico no correlaciona con ninguno de los tres métodos empleados para evaluar la actividad antioxidante. Con base en la medida de la actividad antioxidante para soluciones de ácido ascórbico realizada por el método ABTS y el contenido de este ácido en las diferentes muestras de guayaba fue posible calcular la contribución del ácido ascórbico a la actividad antioxidante total de las muestras. De esta manera, se obtuvieron contribuciones del 1, 3, 3 y 15\% para las guayabas "Pera", "Regional Roja", "Regional Blanca" y "Manzana", respectivamente. Aunque las guayabas en estudio tienen altos niveles de ácido ascórbico, la baja contribución de este ácido a la actividad antioxidante medida por ABTS sugiere que deben existir otros compuestos, incluidos los fenólicos, que contribuyen de manera más importante que el ácido ascórbico en la actividad antioxidante.

Tabla 3. Coeficientes de correlación de Pearson para ácido ascórbico, dehidroascórbico, vitamina $\mathrm{C}$, fenoles totales y actividades antioxidantes

\begin{tabular}{lllllll}
\hline Variable & ADHA & VIT C & FT & ABTS & FRAP & DPPH \\
\hline AA & $0,634^{*}$ & $0,985^{* *}$ & $0,472^{\mathrm{NS}}$ & $-0,001^{\mathrm{NS}}$ & $0,193^{\mathrm{NS}}$ & $0,085^{\mathrm{NS}}$ \\
ADHA & - & $0,759^{* *}$ & $0,862^{* *}$ & $0,331^{\mathrm{NS}}$ & $0,632^{*}$ & $0,451^{\mathrm{NS}}$ \\
VIT C & - & - & $0,591^{*}$ & $0,07^{\mathrm{NS}}$ & $0,305^{\mathrm{NS}}$ & $0,173^{\mathrm{NS}}$ \\
FT & - & - & - & $0,484^{\mathrm{NS}}$ & $0,757^{* *}$ & $0,656^{* *}$ \\
ABTS & - & - & - & - & $0,655^{* *}$ & $0,544^{*}$ \\
FRAP & - & - & - & - & - & $0,840^{* *}$ \\
\hline
\end{tabular}

AA: ácido ascórbico, ADHA: ácido dehidroascórbico, VIT C: vitamina C, FT: fenoles totales. NS: no significativo, ${ }^{*} \mathrm{P}<0,05 ; * * \mathrm{P}<0,01$

De otro lado, el contenido de compuestos fenólicos totales explica la actividad antioxidante medida por los métodos FRAP y DPPH pero no los de ABTS. La no correlación entre ABTS y fenoles puede deberse a la alta dispersión en estas variables obtenida para la guayaba "Pera". De otro lado, se obtuvo correlación entre los métodos ABTS, FRAP y DPPH. 
Dada la amplia variedad de productos elaborados a partir de la guayaba, entre los que se encuentran los néctares, siropes, bocadillos, jaleas, entre otros, es importante profundizar en los efectos que pueden tener los procesos involucrados en la obtención de estos productos sobre el contenido de vitamina $\mathrm{C}$ y la capacidad antioxidante.

\section{CONCLUSIONES}

Los valores obtenidos ubican a las guayabas "Regional Roja", "Regional Blanca" y "Manzana" como fuentes excelentes de vitamina $\mathrm{C}$, en tanto que la guayaba "Pera" tiene un aporte más restringido en esta vitamina. Los contenidos de fenoles totales y la actividad antioxidante medida por ABTS, FRAP y DPPH indican que las guayabas "Pera", "Regional Roja" y "Regional blanca" pueden llegar a contribuir de manera importante en la ingesta de antioxidantes.

\section{AGRADECIMIENTOS}

Los autores agradecen el apoyo económico brindado por el Ministerio de Agricultura y Desarrollo Rural de Colombia, a través del proyecto "Obtención de antioxidantes y fibra dietaria a partir de la guayaba" del programa de investigación "Desarrollo de productos funcionales promisorios a partir de la guayaba para el fortalecimiento de la cadena productiva“. El traslado Cochabamba-Bogotá y sostenimiento de la Ingeniera de Alimentos D. Rojas fue financiado por la Comunidad Económica Europea a través de la "Red química y tecnológica de productos agroindustriales latinoamericanos-lagrotech", red ALFA-LAGROTECH. También se desea agradecer a la Universidad Nacional de Colombia y en especial a la profesora L. P. Restrepo S. por su asesoría.

\section{REFERENCIAS}

1. Temple, N. J.; Nutr. Res. 2000, 20, 449; Lopaczynski, W.; Zeisel, S. H. ; Nutr. Res. 2001, 21, 295; Rush, E.; Ferguson, L. R.; Cumin, M.; Thakur, V.; Karunasinghe, N.; Plank, L.; Nutr. Res. 2006, 26, 197; Ajila, C. M.; Naidu, K. A.; Bhat, S. G.; Prasada, R. U. J. S.; Food Chem. 2007, 105 , 982; Mezadri, T.; Villaño, D.; Fernández-Pachón, M. S.; García-Parrilla, M. C.; Troncoso, A. M.; J. Food Comp. Anal. 2008, 21, 282; Raffo, A.; La Malfa, G.; Fogliano, V.; Maiani, G.; Quaglia, G.; J. Food Comp. Anal. 2006, 19, 11.

2. Patthamakanokporn, O.; Puwastien, P.; Nitithamyong, A.; Sirichakwal, P. P.; J. Food Comp. Anal. 2008, 21, 241

3. Vasco, C.; Ruales, J.; Kamal-Eldin, A.; Food Chem. 2008, 111, 816.

4. Thaipong, K.; Boonprakob, U.; Crosby, K.; Cisneros-Zevallos, L.; Hawkins, B. D.; J. Food Comp. Anal. 2006, 19, 669.

5. Bashir, H. A.; Abu-Goukh, A.-B. A.; Food Chem. 2003, 80, 557.

6. Guo, Ch.; Yang, J.; Wei, J.; Li, Y.; Xu, J.; Jiang, Y.; Nutr. Res. 2003, 23, 1719 .

7. Jimenez-Escrig, A.; Rincón, M.; Pulido, R.; Saura-Calixto, F.; J. Agric. Food Chem. 2001, 49, 5489

8. Gökmen, V.; Kahraman, N.; Demir, N.; Acar, J.; J. Chromogr., A 2000, $881,309$.

9. Hernández, Y.; Lobo, M. G.; González, M.; Food Chem. 2006, 96, 654. 10. Prior, R. L.; Wu, X.; Schaich. K.; J. Agric. Food Chem. 2005, 53, 4290.

11. Singleton, V. L.; Rossi, J. A. Jr.; Am. J. Enol. Vitic. 1965, 16, 144.

12. Re, R.; Pellegrini, N.; Proteggente, A.; Pannala, A.; Yang, M.; RiceEvans, C.; Free Radical Biol. Med. 1999, 26, 1231.

13. Benzie, I. F. F.; Strain, J. J.; Anal. Biochem. 1996, 239, 70

14. Sánchez-Moreno, C.; Larrauri, J. A.; Saura-Calixto, F.; J. Sci. Food Agric. 1998, 76, 270. 